\title{
New analyses of standard physiological signals: the old pipes give the sweetest smoke
}

\author{
Joseph Rinehart • Maxime Cannesson
}

Published online: 22 November 2012

(c) Springer Science+Business Media New York 2012

In this issue of the Journal of Clinical Monitoring and Computing, Giraud et al. investigated the impact of hypovolemia on the respiratory variations in the QRS amplitude on a classical DII EKG lead ( $\triangle$ RDII) [1]. In this animal study, they were able to demonstrate that bleeding and volume expansion induced significant changes in $\Delta$ RDII and that these changes mirrored those observed in the respiratory variations in pulse pressure (PPV). These observations suggest that this non-invasive and potentially widely available parameter could predict fluid responsiveness and detect hypovolemia in operating rooms and intensive care units.

Studies previously published in the Journal of Clinical Monitoring and Computing have already demonstrated a significant relationship between $\triangle$ RDII and PPV (and between $\Delta$ RDII and stroke volume variation) in mechanically ventilated patients under general anesthesia $[2,3]$. The manuscript by Giraud et al. builds on these previous studies by showing that $\Delta$ RDII is also sensitive to manually induced changes in ventricular preload. The remaining steps before acceptance of this new index in clinical practice will be to: (1) show that it can independently predict fluid responsiveness; and (2) that its calculation can be automated and monitored continuously. Thus, while the

\footnotetext{
J. Rinehart · M. Cannesson ( $₫)$ e-mail: mcanness@uci.edu

M. Cannesson

Masimo Corp., Edwards Lifesciences, Covidien,

Mansfield, MA, USA

M. Cannesson

Draeger, Lubeck, Germany
}

Department of Anesthesiology \& Perioperative Care, University of California Irvine, 101 S City Drive, Orange, CA 92868, USA study by Giraud et al. does not yet confirm this new index as an accurate predictor of fluid responsiveness, it is nevertheless a significant step towards validation.

This study exemplifies a new trend in the analysis of physiological signals in which an old and readily available stream of data is processed in a novel way to yield new information. In the present case, the electrocardiogram (EKG) was first described over 100 years ago and has been in clinical use for patient monitoring for decades [4]. Despite this long history, the possible use of the EKG waveform in predicting fluid responsiveness is only now becoming clear. Interestingly, the approach described in the paper by Giraud et al. is very similar to what happened in the analysis of the plethysmographic waveform: both signals have been clinically available for decades but the analysis of their respiratory variations ( $\triangle$ RDII for the EKG and $\triangle \mathrm{POP}$ for the plethysmographic waveform [5]) is a new concept which may have significant clinical utility [6]. The beauty of these new indices (if proven accurate) is that the equipment needed to obtain them is already available at the bedside and that they require very little computing in order to be obtained continuously and automatically. Additionally, they are cheap, and could be used almost anywhere. These old signals may thus provide universal, non-invasive, and inexpensive ways of monitoring fluid responsiveness in a variety of environments (hence the Irish proverb that "the old pipe gives the sweetest smoke").

A similar trend has been observed in cardiac output (CO) monitoring [7]. The traditional clinical approach to monitoring $\mathrm{CO}$ is, of course, transpulmonary thermodilution, and this is still the preferred approach in many settings. The highly invasive nature of pulmonary artery catheters and the associated risk, however, makes them undesirable for routine $\mathrm{CO}$ monitoring across the full spectrum of surgical and intensive care patients. Newer 
measurements of $\mathrm{CO}$ derived from old signals are also stepping into fill this gap. There are a variety of monitors capable of estimating $\mathrm{CO}$ from arterial pressure waveforms, providing a minimally invasive approach [7]. Other monitors calculate $\mathrm{CO}$ from fully non-invasive sources like thoracic impedance or conductance [7]. Taken in whole, the trend of improved monitoring from less invasive sources will continue to make surgery safer. As just one example, application of goal-directed fluid therapy (GDFT) typically requires a measure of stroke volume or $\mathrm{CO}$, and ideally a measure of at least one dynamic predictor of fluid responsiveness. The ability to obtain these values from minimally invasive monitors now allows use of GDFT in a much wider patient population than would otherwise be possible.

While these new indices may have high utility, it is also of paramount importance to remember that extracting information from complex signals has limitations. For example, though $\triangle \mathrm{POP}$ has been consistently shown to be an accurate predictor of fluid responsiveness, one has to remember that the plethysmographic signal is very sophisticated and affected by many physiologic factors [8]. Acute changes in vasomotor tone induced by pain or by any other phenomenon can impact the waveform and the accuracy of $\triangle \mathrm{POP}$. For $\Delta \mathrm{RDII}$ extracted from the EKG waveform, several limitations can already be predicted. Since this signal relies on thoracic impedance, it is highly probable that changes in intrathoracic fluid or air will impact this index. Additionally, it is likely that ventricular dilation, hypertrophy, or other cardiac conduction abnormalities may induce changes in this index since the electric axis of the heart may be impacted by these modifications. Prone position may be a significant limitation to their use, as well. These are just a few obvious examples, but other limitations may arise.

Ultimately, however, if $\triangle \mathrm{RDII}$ is found to not just correlate with PPV but independently predict fluid responsiveness, as now seems plausible, practitioners will have one more tool applicable to a wide population of patients to guide clinical care. Giraud et al. have shown that the EKG, indeed an old "pipe", still has sweet smoke left to give.

Conflict of interests Joseph Rinehart and Maxime Cannesson are co-inventors and co-owners of US patent 13/95,827: "Method and apparatus for assessment of fluid responsiveness using the EKG waveform".

\section{References}

1. Giraud R, Siegenthaler N, Morel DR, Romand JA, Brochard L, Bendjelid K. Respiratory changes in ECG-wave amplitude is a reliable parameter toestimate intravascular volume status. J Clin Monit Comput. 2012. In Press.

2. Cannesson M, Keller G, Desebbe O, Lehot JJ. Relations between respiratory changes in R-wave amplitude and arterial pulse pressure in mechanically ventilated patients. J Clin Monit Comput. 2010;24: 203-7.

3. Soltner C, Dantec R, Lebreton F, Huntzinger J, Beydon L. Changes in R-Wave amplitude in DII lead is less sensitive than pulse pressure variation to detect changes in stroke volume after fluid challenge in ICU patients postoperatively to cardiac surgery. J Clin Monit Comput. 2010;24:133-9.

4. Cooper JK. Electrocardiography 100 years ago. Origins, pioneers, and contributors. New Engl J Med. 1986;315:461-4.

5. Cannesson M, Attof Y, Rosamel P, Desebbe O, Joseph P, Metton $\mathrm{O}$, Bastien O, Lehot JJ. Respiratory variations in pulse oximetry plethysmographic waveform amplitude to predict fluid responsiveness in the operating room. Anesthesiology. 2007;106: 1105-11.

6. Shelley KH. Photoplethysmography: beyond the calculation of arterial oxygen saturation and heart rate. Anesth Analg. 2007;105: S31-6.

7. Funk DJ, Moretti EW, Gan TJ. Minimally invasive cardiac output monitoring in the perioperative setting. Anesth Analg. 2009;108: 887-97.

8. Landsverk SA, Hoiseth LO, Kvandal P, Hisdal J, Skare O, Kirkeboen KA. Poor agreement between respiratory variations in pulse oximetry photoplethysmographic waveform amplitude and pulse pressure in intensive care unit patients. Anesthesiology. 2008;109: 849-55. 